\title{
Descripción de los Aspectos Comerciales del Esquema del Cargo por Confiabilidad en el Mercado Eléctrico Colombiano
}

\author{
Description of the Commercial Components of the Reliability Charge Scheme in the \\ Colombian Electricity Market.
}

\author{
Juan Carlos Bedoya, Edwin Ariel Rodas, Diego Felipe García. \\ Dirección de Intercambios del Mercado, XM Compañía de Expertos en Mercados S.A. E.S.P. \\ Correo-e:jcbedoya@xm.com.co; erodas@xm.com.co; dfgarcia@xm.com.co
}

\begin{abstract}
Resumen- El Cargo por Confiabilidad en Colombia es un esquema de remuneración que permite hacer viable la inversión en los recursos de generación necesarios para garantizar la expansión del parque de generación bajo señales de eficiencia económica para la atención de la demanda de energía eléctrica incluso bajo condiciones críticas de abastecimiento, a través de señales de largo plazo con las cuales se busca la estabilización de los ingresos de los generadores.
\end{abstract}

En este artículo se presenta una descripción de las componentes comerciales del cargo por confiabilidad con el objetivo de describir de manera sencilla el funcionamiento de este esquema y su impacto en el Mercado de Energía Eléctrica Colombiano.

Palabras clave - Cargo por Confiabilidad, Condiciones de Escasez, Mercado de Energía Mayorista.

Abstract - The Reliability Charge is a remuneration scheme that allows investment in sustainable generation resources necessary to ensure the expansion of the generation system under signals of economic efficiency to meet the electricity energy demand even under critical conditions, through long-term signals which seeks to stabilize generators incomes.

This paper provides a description of the commercial components of the Reliability Charge in order to easily describe the operation of this scheme and its impact on Colombian Electricity Energy Market.

Key Word - Reliability Charge, Scarcity Conditions, Energy Market.

\section{INTRODUCCIÓN}

El mercado de Energía Mayorista en Colombia dio inicio el 20 de julio de 1995; este esquema de mercado remunera a los recursos de generación de acuerdo con su eficiencia en precios otorgando a todos unos precios iguales al precio de oferta del último recurso que abastece a la demanda; este esquema de mercado es conocido como mercado de precios marginales y el precio de remuneración de la energía transado en dicho mercado es el precio de bolsa.

Por otra parte, dentro del mercado de energía eléctrico colombiano, se adoptó el modelo del Cargo por Capacidad el cual consistía en un mecanismo de remuneración administrado de la capacidad de generación, que garantizaba un ingreso fijo anual por megavatio instalado, a un precio definido por el regulador; como desventaja dentro de este esquema, se presentaba que no existía una obligación concreta de los generadores, asociada a esta remuneración.

Posteriormente y a partir del mes de diciembre de 2006, entró en vigencia el modelo actual de Cargo por Confiabilidad como un esquema que permite a los generadores contar con un ingreso de dinero fijo, independientemente de su participación diaria en el mercado mayorista, asignado para períodos de hasta 20 años, reduciendo el riesgo de su inversión para el caso construcción de nuevos recursos de generación. Como contraprestación, los generadores deben mantenerse disponibles y cumplir con sus Obligaciones de Energía Firme cuando se presenten las condiciones de escasez en el sistema. Para la asignación y la determinación del precio se utiliza un mecanismo de mercado que propende por la eficiencia: Las subastas de Obligaciones de Energía Firme [1].

En este artículo se presenta una descripción de las componentes comerciales más relevantes del cargo por confiabilidad ( $\mathrm{CxC}$ ): Las Asignaciones de Obligaciones de Energía Firme (OEF), la Remuneración Real Individual Diaria (RRID), el valor del Costo Equivalente y el Costo Equivalente Real de la Energía (CEE y CERE), Precio de Escasez (PE), los Anillos de Seguridad y finalmente las Pruebas de Disponibilidad (PD). Todo este resumen comercial del Cargo por Confiabilidad permite entender de manera sencilla el funcionamiento de este esquema y los impactos que este posee en el mercado de energía mayorista en Colombia. 


\section{ASIGNACIONES DE OEF}

La Obligación de Energía Firme (OEF) es el vínculo resultante del proceso de Asignación, conocido como Subastas del Cargo por Confiabilidad o mecanismo que haga sus veces, que impone a un generador el deber de generar, de acuerdo con el Despacho Ideal, una cantidad diaria de energía, cuando el Precio de Bolsa supere el Precio de Escasez; a esta condición se le conoce como condición de escasez [1].

El propósito de la Asignación de OEF a los generadores consiste en garantizar a futuro, que estos puedan abastecer la demanda de energía eléctrica para un período de un año (período de vigencia de la obligación) bajo condiciones eficientes de precios. En contraprestación, la demanda remunerará a los generadores por este servicio a través de un ingreso fijo con el cual se propende por la estabilización de los ingresos del generador; dicho ingreso será proporcional a cada kWh de OEF asignado al generador. Más adelante en el capítulo III de este artículo se presenta el cálculo detallado de esta remuneración.

La OEF es asignada a cada generador, o participante interesado en la obtención de la misma, a través de uno de tres esquemas de asignación regulados por la Comisión de Regulación de Energía y Gas-CREG, y son:

- Asignación a Prorrata [3]

- Subastas de Reloj Descendente [4]

- $\quad$ Subastas GPPS [5][6]

Cada uno de estos mecanismos permite determinar la OEF que será asignada a cada generador y el precio al cual será remunerada dicha OEF.

El insumo con el cual el generador participa del mecanismo de asignación corresponde con su Energía Firme para el Cargo por Confiabilidad (ENFICC) la cual corresponde con la energía que es capaz de entregar el generador durante un año de manera continua en condiciones de baja hidrología [7].

El resultado del proceso de asignación corresponde con la $\mathrm{OEF}$, la cual posee una duración de al menos un año (OEF Anual). Este período se define como período de vigencia de la OEF; inicia el 1 de diciembre y termina el 30 de noviembre del siguiente año. Para recursos de generación con casos particulares, tales como plantas nuevas, especiales o existentes con obras, el período de vigencia de la OEF puede ser escogido por los mismos participantes por hasta 20, 10 o cinco años respectivamente. Para el caso de plantas nuevas, el período se verá reducido por los años de antiguiedad de fabricación de la turbina y/o el generador los cuales no podrán superar en ningún caso los tres años [12].

\section{A. Asignación de OEF a Prorrata.}

Este es el mecanismo de Asignación de OEF básico y consiste en distribuir una cantidad de energía, equivalente a la demanda objetivo que se desea cubrir mediante OEF, a prorrata de la ENFICC de los participantes [3].

$$
O E F_{r}=\operatorname{Dem}_{O b j_{\text {Sist }}} \frac{E N F I C C_{r}}{\sum_{k \in \text { Conjunto de } r} E^{2} F I C C_{k}}
$$

Donde

$\boldsymbol{O E F}_{\boldsymbol{r}}$ : Obligación de energía firme Anual, del recurso de generación, en kWh-año.

Dem Objsist: Demanda Objetivo del Sistema, que se debe respaldar mediante Asignaciones de Obligación de Energía Firme para el período de vigencia.

ENFICC : Energía Firme para el Cargo por Confiabilidad declarada y verificada por el Centro Nacional de Despacho-CND, para el recurso $\boldsymbol{r}$ durante el período de vigencia a asignar.

En este mecanismo pueden participan recursos de generación, nuevos, especiales, existentes con obras y existentes; cada uno de estos tipos de plantas podrá optar por hasta por 20, 10, 5 y un año de vigencia de la OEF que le sea asignada. A través de este esquema que garantiza el ingreso del generador hasta por 20 años, se busca alcanzar el objetivo de estabilización del ingreso del generador.

Finalmente, el precio de remuneración de la OEF asignada a cada planta, será el precio al cual haya cerrado la última subasta que haya sido realizada, según lo establecido en el artículo 29 de la Resolución CREG 071 de 2006.

\section{B. Subastas de Reloj Descendente}

Este mecanismo de Asignación consiste en distribuir la demanda objetivo a cubrir con OEF por medio de un esquema de subasta de puja de precios a la baja (reloj descendente) mediante el cual se determina el conjunto de recursos de generación más eficientes en precio que son capaces de cubrir a la demanda [4].

A diferencia de la asignación a prorrata, en el proceso de subasta de reloj descendente, la demanda es elástica y se encuentra definida por tres puntos dentro de la curva EnergíaPrecio así: Margen M1, Demanda Objetivo DO, Margen $M 2$ y precios $2 \boldsymbol{C E}, \boldsymbol{C E}$ y $\mathbf{0 . 5 C E}$ respectivamente, tal como se muestra en la figura 1.

Las variables $\boldsymbol{M 1}, \boldsymbol{M 2}$ y $\boldsymbol{D} \boldsymbol{O}$ poseen los descuentos asociados con las Obligaciones de Energía Firme asignadas en subastas previas y la Energía Firme para el cargo por confiabilidad asociada a las plantas no despachadas centralmente que comprometieron su energía a través de contratos de largo 
plazo para el período de vigencia a subastar. La variable $\boldsymbol{C E}$ (Costo del Entrante) representa el precio base por unidad de energía construido; las asignaciones de obligaciones de energía firme resultantes de la subasta no podrán ser remuneradas por encima del doble o por debajo de 0.5 veces el valor del $\boldsymbol{C} \boldsymbol{E}$.

El proceso de puja a la baja se realiza mediante un esquema de rondas a través del cual un subastador fija un intervalo de tiempo y un rango de precios (precio de apertura y precio de cierre de la ronda) para que los participantes realicen la oferta del precio al cual están dispuestos que se les remunere su posible OEF.

En este proceso de subasta podrán participar recursos de generación nuevos, especiales, existentes con obras y existentes; sólo las plantas nuevas y los bloques de energía asociados con las obras a construir de las plantas especiales y/o existentes con obras, podrán realizar ofertas de precio de puja a la baja en cada una de las rondas de la subasta; los recursos clasificados como existentes son tomadores de precio y no podrán realizar ofertas de precios durante el proceso de subasta. Para estos últimos existe un esquema de retiro voluntario dentro del proceso de subasta que les permite salir del proceso a un precio determinado; sin embargo, la información del precio de retiro de plantas existentes debe ser declarada por el participante meses antes del inicio del proceso de subasta.

En las dos subastas de reloj descendente que ha habido para la asignación de OEF en el mercado eléctrico colombiano, realizadas en junio de 2008 y diciembre de 2011, los períodos de vigencia a subastar han sido Dic2012-Nov2013 y Dic2015Nov2016 respectivamente. Como se puede observar el período de anticipación con el cual se ha asignado la OEF corresponde en ambos casos con cuatro años. A este período de anticipación se le denomina periodo $\boldsymbol{p}$ (Período de Planeación de la Subasta). Lo anterior implicó que los recursos de generación nuevos, especiales o existentes con obras que hubiesen participado dentro de este mecanismo de asignación, debieron estar en operación comercial por lo menos dentro de los cuatro años siguientes a la realización del proceso de asignación de OEF.

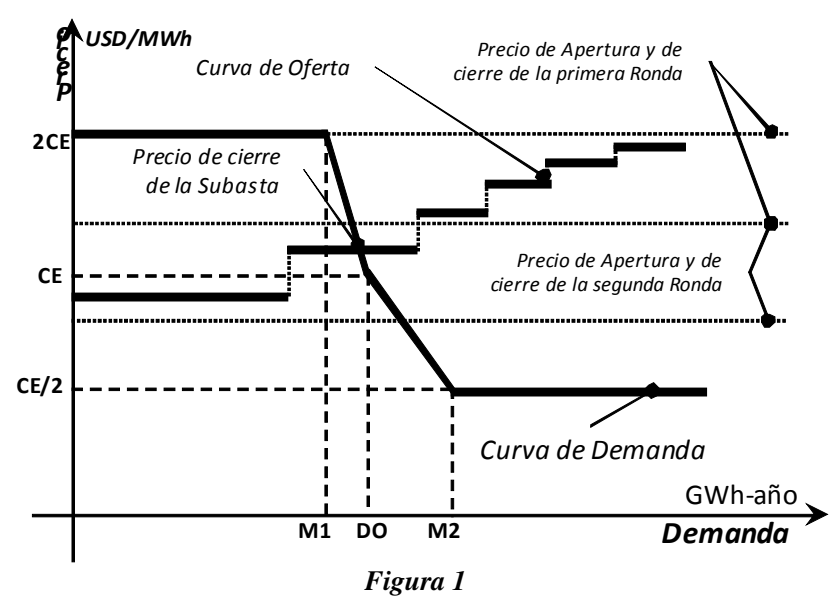

Curva de Oferta y Demanda de la Subasta de Reloj Descendente. Se destaca el esquema de Rondas, el precio de cierre y los parámetros de la curva de demanda de la Subasta

\section{B. Subastas GPPS}

La subasta para recursos de generación cuyo período de construcción es superior al período de planeación de la subasta (GPPS), corresponde con el proceso mediante el cual se asignan OEF a un grupo particular de generadores cuyo proceso de construcción es menor a 10 años, pero no se ajusta al período de planeación definido para las subastas de reloj descendente.

Por ejemplo, se mostró anteriormente que para las dos subastas de reloj descendente que se han realizado en el mercado eléctrico colombiano, el período de planeación $\boldsymbol{p}$ fue definido como cuatro años; sin embargo, para aquellos generadores cuyo proceso de construcción tomaba más de cuatro años y menos de diez años, se llevó a cabo una subasta GPPS. En la historia del mercado eléctrico colombiano, las subastas GPPS han sido llevadas a cabo en el mes siguiente al mes en el que se realizó la subasta de reloj descendente.

En las dos subastas GPPS mencionadas anteriormente (realizadas en julio de 2008 y enero de 2012), se subastaron los porcentajes de incrementos de demanda de los años Dic2013 a Nov2019 y Dic2016 a Nov2022 para la primera y segunda subasta GPPS respectivamente; estos años corresponden con los años $\boldsymbol{p}+\boldsymbol{1}$ hasta el año $\mathbf{1 0}$.

La subasta GPPS consiste de una subasta de sobre cerrado mediante la cual los generadores ofertan el precio al cual están dispuestos a recibir la remuneración de sus OEF; para efectos del problema de optimización, en caso de que dos o más participantes hayan ofertado el mismo precio, se realiza un desempate entre los precios empatados restando 1USD/GWh de manera aleatoria y posteriormente este último precio es diferenciado para cada uno de los años $\boldsymbol{p}+\boldsymbol{1}$ hasta el año 10 .

$$
p_{r, a}^{i i}=(1-0.000001 a) p_{r}^{i}+0.1 a
$$


Donde:

a: Cada uno de los años de vigencia de las OEF a asignar en la subasta GPPS. Desde p+1 hasta 10.

$p_{r}^{i}:$ Precio ofertado y desempatado.

$\boldsymbol{p}_{\boldsymbol{r}, \boldsymbol{i}} \boldsymbol{i}$ : Precio ofertado, desempatado y diferenciado para cada año a.

Asimismo, los generadores también ofertan los valores mínimo y máximo del rango de OEF por el cual desean ser asignados, es decir, al generador debe asignársele, entre el año $p+1$ y el año 10, un valor de OEF que se encuentre entre los valores mínimo y máximo ofertados, en caso contrario el valor asignado debe ser nulo.

Finalmente, el proceso de asignación es llevado a cabo a través de un proceso de optimización mediante el cual se garantiza la minimización de los costos asociados con la remuneración de las OEF asignadas, sujeto a que la totalidad de OEF asignada debe ser menor que la cantidad máxima de energía a asignar y que las OEF a asignar a cada participante debe encontrarse entre los valores máximo y mínimo ofertados o en su defecto nula.

$\operatorname{Min} \sum_{a=p+1}^{10}\left(\sum_{r \in G P P S} O E F_{r, a} \cdot p_{r, a}^{i i}\right)+1.5 P M G P P S \sum_{a=p+1}^{10}\left(C M_{a}-\sum_{r \in G P P S} O E F_{r, a}\right)$

Sujeto a:

$$
\begin{aligned}
& u_{r} \cdot \operatorname{Emin}_{r} \leq \sum_{a=p+1}^{10} \mathrm{OEF}_{\mathrm{r}, \mathrm{a}} \leq u_{r} \cdot \operatorname{Emax}_{r} \forall r \\
& \sum_{r \in G P P S} O E F_{r, a} \leq C M_{a} \forall a
\end{aligned}
$$

Donde:

\section{r: Recurso de Generación GPPS}

p: Período de Planeación.

GPPS: Conjunto de Plantas GPPS que participan en la subasta de sobre cerrado.

$\boldsymbol{O E F}_{r, a}$ : Obligación de energía Firme a Asignar al recurso $r$ en el año a. Corresponde con la variable de decisión del problema de optimización.

PMGPPS: Precio máximo de la asignación para plantas GPPS. Corresponde con el precio de cierre de la última subasta de reloj descendente realizada con anterioridad a la subasta GPPS.

CMa: Cantidad máxima de energía a asignar en el año a. La cantidad máxima a asignar se calcula como se muestra en [5].

Emin: Valor mínimo de la ENFICC a asignar a la planta, ofertada para la subasta de sobre cerrado.

Emax: Valor máximo de la ENFICC a asignar a la planta, ofertada para la subasta de sobre cerrado.

\section{REMUNERACIÓN REAL INDIVIDUAL}

La Remuneración Real Individual (RRID) es el concepto a través del cual se remunera, en el mercado de energía colombiano, a los generadores que cubren la demanda a través de Obligaciones de Energía Firme que le hayan sido asignadas por medio de uno de los mecanismos expuestos en el capítulo II. Esta remuneración es conocida como Remuneración Individual Diaria-RRID y se tienen en cuenta dentro del proceso de cálculo las obligaciones de energía firma diarias y el precio al cual fueron pactadas; adicionalmente, con el objetivo de buscar el cumplimiento comercial de dichas obligaciones, se involucra dentro del proceso de cálculo la disponibilidad comercial de la planta y los respaldos asociados a los diferentes anillos de seguridad del cargo por confiabilidad expuestos en el capítulo VI.

Esta remuneración es pagada al agente generador por la disponibilidad de sus activos de generación que garanticen el cumplimiento de la Obligación de energía firme que le fue asignada en la subasta o el mecanismo que haga sus veces, para asegurar a los usuarios la confiabilidad de la prestación del servicio bajo condiciones críticas.

A continuación, se presenta la fórmula de la remuneración de las obligaciones de energía firme:

$$
R R I D_{r, d}=P C C_{r, d} \cdot O E F_{r, d} \cdot \min \left(1, \frac{O E F V_{r, d}+C C_{r, d}+D D V V_{r, d}+\sum_{h=1}^{24} D C_{r, h}}{O E F_{r, d}+V C_{r, d}}\right)
$$

Donde

$\boldsymbol{R R I D}_{r, d}$ Remuneración Real Individual Diaria del recurso $\boldsymbol{r}$ para el día d, en \$ (se convierte a pesos con la Tasa de cambio Representativa del Mercado-TCRM del último día del mes que contiene al día d).

$\boldsymbol{O E F}_{r, d}$ : Obligación de energía firme diaria del recurso de generación, en kWh-día.

$\boldsymbol{P C C}_{\boldsymbol{r}}$ : Precio promedio de remuneración de las obligaciones de energía firme del cargo por confiabilidad, asignadas al recurso de generación $\boldsymbol{r}$ para el día d, en $\$ / k W h$.

$\boldsymbol{O E F V} \boldsymbol{V}_{r, d}$ Obligación de Energía Firme de Venta, adquirida para el recurso de generación, en subastas de reconfiguración de venta, en kWh-día. Corresponde con uno de los anillos de seguridad del Cargo por Confiabilidad.

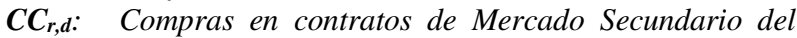
Cargo por Confiabilidad, en $\mathrm{kWh}$. Corresponde con uno de los anillos de seguridad del Cargo por Confiabilidad.

$D D V V_{r, d}:$ Compas en contratos de Mercado Secundario del Cargo por Confiabilidad, en kWh. Corresponde con uno de los anillos de seguridad del Cargo por Confiabilidad.

DC $\boldsymbol{r}, \boldsymbol{h}$ : Disponibilidad Comercial del Recurso para cada hora del día, en $\mathrm{kWh}$.

$\boldsymbol{V C}_{\boldsymbol{r}, \boldsymbol{d}}$ : Ventas en contratos de Mercado Secundario del Cargo por Confiabilidad, en $\mathrm{kWh}$. 
Para efectos de la liquidación y facturación del cargo por confiabilidad, se calcula el valor de la RRI mensual del agente mediante la agrupación del RRID de las plantas que el agente generador representó durante cada uno de los días del mes. Posteriormente, se calcula la Remuneración Real Total del Sistema, como la suma del RRI mensual de cada agente.

$$
R R T_{m}=\sum R R I_{A, m}
$$

Donde

$\boldsymbol{R R T}_{\boldsymbol{m}}: \quad$ Remuneración Real Total del Sistema para el mes $\boldsymbol{m}$, en $\$$.

$\boldsymbol{R R I}_{A, m}: \quad$ Remuneración Real Individual del Agente A, para el mes $\boldsymbol{m}$.

\section{A. Asignación de Obligación de Energía Mensual.}

Tal como se describió en el capítulo II, las asignaciones de OEF se realizan para un período de vigencia anual, sin embargo, teniendo en cuenta las reglas del mercado definidas en la Resolución CREG 071 de 2006 y las demás reglas que definen las transacciones comerciales, la liquidación de los diferentes conceptos del mercado de energía Colombiano se realizan en un horizonte de tiempo horario, diario y mensual.

Según lo anterior, es necesario convertir los valores de OEF asignada a cada recurso de generación a un período mensual. El valor mensual se obtiene mediante la demanda objetivo definida por el regulador mes a mes para el periodo de vigencia de la obligación en curso, así:

$$
O E F_{r, m}=O E F_{r, a} \frac{\text { DemObj }_{m}}{\operatorname{DemObj}_{a}}
$$

Donde

$$
\begin{array}{cl}
\boldsymbol{O E F}_{r, m}: & \begin{array}{l}
\text { Obligación de Energía Firme del mes } \boldsymbol{m} \text {, para el } \\
\text { recurso de generación } \boldsymbol{r} .
\end{array} \\
\boldsymbol{O E F}_{r, a}: & \begin{array}{l}
\text { Obligación de Energía Firme del período de } \\
\text { vigencia anual a, para el recurso de generación } \boldsymbol{r} .
\end{array} \\
\text { DemObj }_{\boldsymbol{m}}: & \begin{array}{l}
\text { Demanda Objetivo del mes } \boldsymbol{m} \text { del período de } \\
\text { vigencia anual a. }
\end{array} \\
\text { DemObja: } & \text { Demanda Objetivo del período de vigencia anual }
\end{array}
$$
a.

\section{B. Asignación de Obligación de Energía Diaria.}

Tal como se describió anteriormente, en cuanto a las reglas que definen las transacciones comerciales, la liquidación de los diferentes conceptos del mercado de energía Colombiano se realizan en un horizonte de tiempo diario y horario, por lo cual para efectos de la liquidación que realiza el Administrador del Sistema de Intercambios Comerciales-
ASIC, es necesario calcular la Obligación de Energía Firme en valores diarios; para ello, se utiliza la relación entre la demanda comercial diaria y la demanda comercial mensual, teniendo en cuenta la Demanda Desconectable Voluntaria Verificada (capítulo VI), así:

$$
O E F D_{r, d}=O E F_{r, m} \frac{D C_{d}+D D V V_{d}}{D C_{m}+D D V V_{m}}
$$

Donde
OEFD $_{r, m}$ : Obligación de energía firme mensual del recurso de generación, en kWh-mes.
DC $C_{d}$ Demanda Comercial del Sistema para el día d del mes $\boldsymbol{m}$.
DC $\boldsymbol{C}_{\boldsymbol{m}}$ : Demanda Comercial del Sistema para el mes $\boldsymbol{m}$.
DDVVd: Demanda Desconectable Voluntaria y Verificada del sistema para el día d.del mes $\boldsymbol{m}$.
DDV $\boldsymbol{V}_{\boldsymbol{m}}$ : Demanda Desconectable Voluntaria y Verificada del sistema para el mes $\boldsymbol{m}$.

Es importante tener presente que para un mismo período de vigencia, un mismo recurso de generación puede poseer más de una obligación de energía firme asignada a precios diferentes, por lo cual la OEF anual, mensual y diaria del recurso de generación se constituye como la suma de las obligaciones individuales adquiridas en cada una de las subastas.

\section{Precio de Remuneración Diario.}

Dentro de la fórmula para el cálculo de la Remuneración Real Individual Diaria $\boldsymbol{R} \boldsymbol{R} \boldsymbol{I D}$, se presentó la variable $\boldsymbol{P C} \boldsymbol{C}$, la cual corresponde con el precio unificado de las obligaciones de energía firme que le hayan sido asignadas al recurso de generación en una o varias subastas, así:

$$
P C C_{r, d}=\frac{\sum_{S \in \text { Subastas }} O E F_{r, S, d} \cdot P_{r, S}}{\sum_{S \in \text { Subastas }} O E F_{r, S, d}}
$$

Donde

$$
\begin{aligned}
\boldsymbol{O E F}_{\boldsymbol{r}, \boldsymbol{S}, \mathrm{d}}: & \begin{array}{l}
\text { Obligación de energía firme diaria del recurso de } \\
\text { generación } \boldsymbol{r} \text { adquirida en la Subasta } \boldsymbol{S} \text {, en } k W h- \\
\text { día. }
\end{array} \\
\boldsymbol{P}_{\boldsymbol{r}, \boldsymbol{S}}: & \begin{array}{l}
\text { Precio de cierre de la subasta } \boldsymbol{S} \text {, o del mecanismo } \\
\text { que haga sus veces, al cual se remunerarán las }
\end{array} \\
& \text { Obligaciones de Energía Firme asignadas al } \\
& \text { recurso } \boldsymbol{r} \text { en dicha subasta. }
\end{aligned}
$$

En resumen, a través del cálculo de la OEF mensual y la OEF diaria se determina la remuneración de las obligaciones de energía firme del cargo por confiabilidad en el mercado de energía mayorista colombiano; a través del cálculo del Precio de Remuneración de las Obligaciones de Energía Firme, es posible determinar la Remuneración del Cargo por Confiabilidad. 


\section{COSTO EQUIVALENTE REAL DE ENERGÍA}

El esquema del cargo por confiabilidad, tiene como incentivo la expansión del parque de generación en el país, con el objetivo de garantizar el suministro continuo de electricidad bajo el crecimiento continuo de la demanda de manera confiable, segura y económica aún en condiciones críticas, razón por la cual la demanda eléctrica debe incorporar en su tarifa el costo que conlleva la implementación de este esquema. Esta tarifa recibe el nombre de Costo Equivalente Real de Energía (CERE), el cual es calculado mensualmente como se presenta en la ecuación 11. Este valor es incluido en la base de los precios de oferta que presentan los generadores para el despacho económico, e impacta directamente los precios de bolsa que resultan en el despacho ideal, los precios de reconciliación, AGC, etc.

$$
\operatorname{CERE}_{m}=\frac{R R T_{m}}{\sum_{j \in D C} G R_{j, m}+\sum_{k \in N D C} V B_{k, m}}
$$

Donde

$$
\begin{aligned}
& \boldsymbol{C E R E}_{\boldsymbol{m}}: \text { Costo Equivalente Real de la Energía para el mes } \\
& \boldsymbol{m} . \\
& \boldsymbol{G R}_{\boldsymbol{j}, \boldsymbol{m}}: \begin{array}{l}
\text { Generación Real del mes } \boldsymbol{m} \text {, de la planta } \\
\text { despachada centralmente } \boldsymbol{j} .
\end{array} \\
& \boldsymbol{V} \boldsymbol{B}_{\boldsymbol{k}, \boldsymbol{m}}: \quad \begin{array}{l}
\text { Venta en Bolsa del mes } \boldsymbol{m} \text {, de la planta No } \\
\text { despachada centralmente } \boldsymbol{k} .
\end{array}
\end{aligned}
$$

El valor del $\boldsymbol{C E R} \boldsymbol{E}$ sólo puede ser calculado una vez se haya consolidado el valor mensual de las variables $\boldsymbol{R} \boldsymbol{R} \boldsymbol{T}, \boldsymbol{G R}$ y $\boldsymbol{V B}$, es decir que sólo puede ser calculado una vez haya finalizado el mes $\boldsymbol{m}$, por lo tanto, para que cada generador pueda incluir

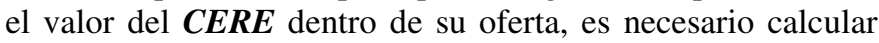
un valor preliminar definido como Costo Equivalente de Energía (CEE); para ello se emplea el escenario medio mensual de proyección de demanda realizado por la Unidad de Planeación Minero Energética (UPME)

$$
C E E_{m}=\frac{\sum_{r \in G} \sum_{S \in \text { Subasta }} O E F_{r, S, m} \cdot P_{r, S}}{E T D P_{m}}
$$

Donde

$\boldsymbol{C E E}_{\boldsymbol{m}}: \quad$ Costo Equivalente de la Energía para el mes $\boldsymbol{m}$. $\boldsymbol{O E F}_{\boldsymbol{r}, \boldsymbol{S}, \boldsymbol{m}:}$ Obligación de Energía Firme del mes $\boldsymbol{m}$, asignada al recurso de generación $\boldsymbol{r}$ en la subasta $\boldsymbol{S}$.

$\boldsymbol{E T D P}_{\boldsymbol{m}}$ : Energía Total Demanda Proyectada para el mes $\boldsymbol{m}$. Tomado del Escenario medio de proyección de la demanda realizado por la UPME.

\section{PRECIO DE ESCASEZ}

El precio de escasez es una de las variables más importantes dentro del esquema de cargo por confiabilidad y del mercado de energía mayorista dado que determina el nivel del precio de bolsa a partir del cual se hacen exigibles las Obligaciones de Energía Firme de un generador y constituye un seguro para la demanda, siendo éste el precio máximo al que la demanda pagará por la energía.

Este valor representa los costos de suministro y transporte de combustible (parte combustible), los costos de operación y mantenimiento y los costos variables en los que incurre una planta de generación térmica operando con combustibles líquidos de origen fósil. El valor del precio de escasez es un valor mensual fijo y actualizado por el ASIC mediante indicadores macroeconómicos.

$$
P E_{m}=P E_{m-1}^{P C}+C O M_{m-1}+O C V_{m-1}
$$

Donde:

$\boldsymbol{P E}_{\boldsymbol{m}}: \quad$ Precio de Escasez del mes $\boldsymbol{m}$.

$\boldsymbol{P E}^{\boldsymbol{P C}} \boldsymbol{m}_{m-1}: \quad$ Precio de Escasez, parte combustible, del mes $\boldsymbol{m}-\mathbf{1}$. COM $_{m-1}$ : Costo de Operación y Mantenimiento del mes m-1. OCV $\boldsymbol{V}_{\mathbf{m}-1}: \quad$ Otros Costos Variables del mes m-1.

Actualmente, el valor del precio de escasez del mes $\boldsymbol{m}$ es calculado dos días calendario antes del inicio de dicho mes.

La componente denominada precio de escasez parte combustible corresponde con los costos de suministro y transporte de combustible. El valor base corresponde con 101,6 US\$/MWh; fue actualizado hasta el año 2010 con el promedio aritmético mensual del Índice diario New York Harbor Residual Fuel No.6 1.0\% Sulfur; posteriormente con la entrada en vigencia de la Resolución CREG 162 de 2010, la actualización del precio de escasez se realiza con el promedio aritmético mensual del Precio de Cierre diario del Índice Platts US Gulf Coast Residual Fuel No.6 1.0\% Sulfur.

$$
P E_{m-1}^{P C}=P E_{m-2}^{P C} \frac{\text { Promedio } \mathrm{Mes}_{m-1}\left(\text { Indice }_{d}\right)}{{\text { Promedio } \mathrm{Mes}_{m-2}\left(\text { Indice }_{d}\right)}^{P C}}
$$

La información de los Costos de Operación y Mantenimiento y los Costos Variables corresponden con los valores de la última facturación realizada por ASIC al momento del cálculo del precio de escasez.

Para enero de 2014, el precio de escasez ascendió a los $450 \$ / \mathrm{kWh}$. 


\section{ANILLOS DE SEGURIDAD DEL CARGO POR CONFIABILIDAD}

Los Anillos de Seguridad del cargo por confiabilidad son un conjunto de mecanismos orientados a facilitar el cumplimiento de las OEF por parte de los generadores. Estos esquemas le permiten al generador desde respaldar su propia indisponibilidad por efectos de mantenimientos y otras causales, hasta honrar las OEF para retrasos (no constituidos como graves e insalvables) en el proceso de construcción de plantas nuevas, especiales o existentes con obras [2].

Los anillos de seguridad fueron concebidos desde el inicio del cargo por confiabilidad, pero el detalle de los mismos se ha venido definiendo en regulación expedida por la Comisión de Regulación de Energía y Gas, durante los últimos años así:

\section{A. Mercado Secundario de Energía Firme del Cargo por Confiabilidad}

El Mercado Secundario de Energía Firme (MSEC) es el mecanismo que le permite a cada uno de los generadores que determinen que su energía no es suficiente para cumplir sus Obligaciones de Energía Firme, negociar a través de un contrato bilateral, un respaldo adicional de energía con otros generadores cuyo único destino es el de honrar OEF del comprador [9].

A las negociaciones del MSEC donde interactúan dos plantas pertenecientes a diferentes agentes del mercado de energía mayorista, se le conoce como Contrato de Respaldo de OEF en el Mercado Secundario, pero cuando las dos plantas pertenecen a un mismo agente del mercado, la transacción se le conoce como Declaración de Respaldo de OEF en el MSEC.

En este mercado los vendedores sólo podrán ser aquellas plantas de generación que posean Energía de Referencia suficiente para el Mercado Secundario, la cual se calcula como sigue:

Para plantas hidráulicas: La Energía Disponible Adicional más la diferencia entre la ENFICC declarada y la ENFICC comprometida, según lo definido en el Artículo 43 de la Resolución CREG 071 de 2006.

Para plantas térmicas: La correspondiente a la diferencia entre la ENFICC y la ENFICC comprometida. En este caso la energía que resulte de esta diferencia debe respaldarse con los contratos de suministro y transporte de combustibles en las mismas condiciones exigidas a la ENFICC asociada a las Obligaciones de Energía Firme, según lo definido en el Artículo 43 de la Resolución CREG 071 de 2006.

Tal como se describió en el capítulo III, las compras y las ventas de energía en el MSEC son empleadas para el cálculo del RRID; los valores a tener en cuenta en dichos cálculos corresponden a las cantidades de energía pactadas entre las partes ya sea a través de un contrato o una declaración de respaldo.

Por otra parte, bajo condiciones de escasez (Precio de Bolsa supera el Precio de Escasez) los valores de compras o ventas en el MSEC a tener en cuenta dentro del cálculo del RRID de una planta, corresponderán con el valor mínimo entre los excedentes de generación ideal del vendedor, la energía requerida para respaldar las OEF del comprador y la cantidad pactada en el contrato de MSEC. Para calcular estos valores se debe tener presente la prioridad en los contratos o declaraciones de respaldo al momento del registro de los mismos ante el Administrador del Sistema de Intercambios Comerciales-ASIC.

\section{B. Demanda Desconectable Voluntaria}

La Demanda Desconectable Voluntaria DDV es el mecanismo que le permite al generador respaldar su OEF a través de uno o varios usuarios que están dispuestos a reducir su demanda a cambio de una contraprestación, a través de la desconexión del consumo de energía eléctrica desde el Sistema Interconectado Nacional (SIN).

Para llevar a cabo este tipo de respaldo se debe llevar a cabo una transacción bilateral entre el agente generador que representa a la planta de generación interesada en respaldar sus OEF con DDV y el agente comercializador que represente al usuario ante el Mercado de Energía Mayorista como un usuario o frontera del tipo DDV [11].

Las fronteras DDV pueden ser del tipo:

Frontera DDV con Línea Base de Consumo: Son aquellas fronteras en las que el consumo de los usuarios tiene frecuencia y poca variabilidad y que corresponden a las que tienen un error no mayor al 5\% respecto a la estimación efectuada con el modelo presentado en el anexo 1 de [11].

Frontera DDV con medición Directa: Son aquellas en las cuales se puede determinar la cantidad de energía que fue desconectada del SIN a través de un proceso de medición; estas se clasifican fronteras DDV con planta de emergencia en la cual el usuario se apoya de una planta auxiliar que lo alimenta durante la desconexión para así disminuir o suprimir los requerimientos de energía del SIN y las fronteras DDV con medición independiente en las cuales el usuario tiene definido el consumo de un proceso de producción que utiliza diariamente y puede desconectarlo en cualquier momento.

De la misma manera que los Contratos de MSEC, el valor de la energía asociada con la Demanda Desconectable Voluntaria impacta de manera directa el valor de la RRID del generador. 


\section{Plantas de Generación de última instancia (GUI)}

Este mecanismo fue regulado mediante la Resolución CREG 153 de 2011; permite a cada uno de los generadores que determinen que su energía no es suficiente para cumplir sus OEF, respaldarlas por medio de plantas GUI (se exceptúan los autogeneradores y cogeneradores) que se encuentran en operación al momento de hacer uso de este Anillo de Seguridad. Las plantas GUI pueden ser de dos tipos:

- Construidas que no están conectadas al SIN.

- Por construir o instalar.

Las plantas GUI deberán ser despachadas centralmente y sus costos variables de combustible estimados no podrán ser superiores a la componente Parte Combustible (PC) del Precio de Escasez (PE)

\section{Subastas de Reconfiguración de Venta}

Las Subastas de Reconfiguración son el mecanismo mediante el cual se puede ajustar un exceso o el déficit de cobertura de Obligaciones de Energía Firme por cambios en las proyecciones de demanda de energía con respecto a la demanda con la cual se realizó la Asignación de OEF. Este esquema fue regulado mediante la Resolución CREG051 de 2012. Si bien existen las Subastas de Reconfiguración tanto de Compra (SRCFC) como de venta (SRCFV), son estas últimas las que se constituyen como un anillo de seguridad para aquellos generadores que estiman que su energía no será suficiente para respaldar sus $\mathrm{OEF}$.

En el esquema de SRCFV participan los generadores como compradores de las Obligaciones de Energía Firme de Venta (OEFV) a través de un esquema de subasta de sobre cerrado mediante la cual los generadores ofertan el precio máximo al cual están dispuestos a comprar los derechos correlativos a las $\mathrm{OEF}$ previamente asignadas. Asimismo, los generadores también ofertan los valores mínimo y máximo de OEFV que desean adquirir es decir, que al generador se le asignaría un valor nulo de OEFV en caso de no ser posible, bajo los criterios de la SRCFV, asignarle un valor de OEFV que se encuentre entre el valor mínimo de y máximo de OEFV ofertados por el participante.

Finalmente, el proceso de asignación es llevado a cabo a través de un proceso de optimización mediante el cual se garantiza la maximización del valor de la venta de los derechos correlativos de OEF asociada con la compra de OEFV que realizan los participantes de la SRCFV, sujeto a que la totalidad de la OEFV asignada debe ser menor que la cantidad máxima de OEFV a asignar y que los valores de OEFV a asignar a cada participante debe encontrarse entre los valores máximo y mínimo ofertados o en su defecto deberá ser nulo.

$$
\operatorname{Min} \sum_{r \in S R C F V} O E F V_{r} \cdot P s r c f v_{r}^{i}
$$

Sujeto a:

$$
\begin{aligned}
& u_{r} \cdot \operatorname{Omin}_{r} \leq \mathrm{OEFV}_{\mathrm{r}} \leq u_{r} \cdot \operatorname{Omax}_{r} \quad \forall r \\
& \sum_{r \in S R C F V} O E F V_{r} \leq C M
\end{aligned}
$$

Donde:

r: Recurso de Generación participante de la Subasta de reconfiguración de Venta-SRCFV.

SRCFV: Subasta de Reconfiguración de Venta para la Asignación de Obligaciones de energía Firme de Venta.

Psrcfvir: Precio Ofertado para el recurso de generación $\boldsymbol{r}$, para la compra de Obligaciones de Energía Firme de Venta OEFV. Este precio es desempatado mediante un proceso aleatorio en caso de que dos o más recursos hayan ofertado el mismo valor.

OEFV $:$ Obligación de energía Firme de Venta a Asignar al recurso $\boldsymbol{r}$. Corresponde con la variable de decisión del problema de optimización.

CM: Cantidad máxima de energía a asignar en Obligaciones de Energía Firme de Venta.

Omin: Obligación de Energía Firme de Venta mínima a asignar al recurso de generación $\boldsymbol{r}$ en la subasta de reconfiguración de venta.

Omax: Obligación de Energía Firme de Venta máxima a asignar al recurso de generación $\boldsymbol{r}$ en la subasta de reconfiguración de venta.

\section{PRUEBAS DE DISPONIBILIDAD DEL CARGO POR CONFIABILIDAD}

Las Pruebas de Disponibilidad del Cargo por Confiabilidad (PD) son un mecanismo mediante el cual se valida la disponibilidad física de una planta de generación con OEF asignada a través de un proceso de selección aleatorio en el cual se programa a la planta para que inyecte energía al sistema interconectado nacional (SIN) bajo condiciones particulares de capacidad y duración establecidas por la Regulación.

Las PD del Cargo por Confiabilidad fueron inicialmente reglamentadas por la Resolución CREG 085 de 2007 y fueron modificados posteriormente por las Resoluciones CREG 138 de 2008, CREG 177 de 2008, CREG 138 de 2009, CREG 148 de 2010, CREG 138 de 2012 y finalmente CREG 154 de 2013.

El proceso aleatorio que compete a las PD consiste básicamente en que todos los días, para cada planta de generación que no salga programada para generar en el despacho económico, el Centro Nacional de Despacho (CND) calcula la probabilidad de que dicha planta sea llamada a prueba tal como se muestra a continuación: 


$$
P_{g}=\mid \begin{array}{cc}
\frac{1}{30} & M_{g}>12 \\
\frac{1}{30 \times\left(12-M_{g}+1\right)} & 12 \geq M_{g}>0 \\
\frac{1}{30 \times(12) \times 2} & M_{g}=0
\end{array}
$$

Donde

Mg: Número de meses consecutivos anteriores a la fecha de cálculo, contados desde el último mes en que la planta haya tenido generación real [10].

Se exceptúan de la programación de las pruebas de disponibilidad, aquellos casos en los que la planta de generación se encuentre aislada del SIN, o cuando por requerimientos de seguridad y confiabilidad del SIN, o por aplicación del Decreto 880 de 2007, no pueda ser despachada en ningún período [10].

La duración de la prueba será de 4 horas consecutivas y se debe garantizar que el inicio y la finalización de la misma ocurran dentro del mismo día; el valor de generación programado para la prueba deberá ser igual a la declaración de disponibilidad realizada para la planta para cada uno de los períodos en los que fue programada la PD y para el caso de plantas térmicas, el combustible que la planta deberá emplear para la realización de la prueba deberá ser el combustible declarado para el despacho económico.

Los recursos de generación a los que se les programa la PD podrán desviarse del programa de generación en un margen de hasta un $5 \%$ y la prueba será considerada como exitosa cuando la planta tenga una generación total durante el período de la prueba, igual o superior a la generación objetivo considerando la desviación permitida (5\%). En este caso, la remuneración de la energía inyectada al SIN por la planta durante la PD será remunerada al Precio de Reconciliación positiva del que trata la Resolución CREG 034 de 2001.

En el escenario en el cual la prueba de disponibilidad no sea exitosa el agente que representa la planta de generación podrá solicitar que se repita la prueba dentro del mismo día o dentro de los tres días siguientes; si la prueba es exitosa dentro de este plazo, la remuneración de la energía inyectada al SIN por la planta de generación en cada uno de los intentos de la PD será remunerada al precio de bolsa nacional [10].

En el caso de que la repetición de la PD no sea exitosa o en caso de que no se solicite la repetición de la PD dentro de los tres días posteriores a la programación inicial, la planta de generación verá reducidos sus ingresos por la cesación de los pagos correspondientes a la OEF respaldada por la planta (RRID) desde el último día en el cual la planta haya tenido una generación real mayor que cero durante al menos cuatro horas consecutivas y hasta tanto no se presente una PD exitosa; no obstante, el recurso de generación podrá recibir la remuneración asociada únicamente a la porción de su OEF que haya sido respaldada en anillos de seguridad.

\section{CONCLUSIONES}

En este artículo se presentó una descripción sencilla y cronológica del funcionamiento del cargo por confiabilidad y los aspectos comerciales involucrados en el cubrimiento de la demanda a través de Obligaciones de Energía Firme.

Se inició describiendo los procesos de asignación, continuando con los esquemas para la liquidación y el recaudo de la remuneración del cargo por confiabilidad; asimismo se abordaron temas adjuntos al cargo por confiabilidad complementarios a este esquema, tales como los anillos de seguridad y las pruebas de disponibilidad.

Por último, se describió la señal de escasez del mercado de energía mayorista colombiano definida como el precio de escasez.

\section{REFERENCIAS}

[1] CREG, Cargo por Confiabilidad. Esquema Regulatorio para asegurar la confiabilidad en el suministro de energía eléctrica en Colombia. Comisión de Regulación de Energía y Gas CREG. 2006

http://www.creg.gov.co/cxc/download/documentos/C argoxConfiabilidad.pdf

[2] CREG, Resolución CREG061 de 2007. Por la cual se expiden normas sobre las garantías para el Cargo por Confiabilidad.

[3] CREG, Resolución CREG 071 de 2006. Por la cual se adopta la metodología para la remuneración del Cargo por Confiabilidad en el Mercado Mayorista de Energía. Artículo 25. Reglas para la asignación de Obligaciones de Energía Firme para los casos en los cuales no se requiera la realización de una Subasta. Modificado por la Resolución CREG 019 de 2008.

[4] CREG, Resolución CREG 071 de 2006. Por la cual se adopta la metodología para la remuneración del Cargo por Confiabilidad en el Mercado Mayorista de Energía. Anexo 10. Reglamento de la Subasta para la Asignación de Obligaciones de Energía Firme. Modificado por la Resolución CREG019 de 2008, CREG030 de 2008, CREG042 de 2008, CREG139 de 2011 y CREG183 de 2011.

[5] CREG, Resolución CREG 071 de 2006. Por la cual se adopta la metodología para la remuneración del 
Cargo por Confiabilidad en el Mercado Mayorista de Energía. Anexo 11. Procedimiento para Asignación de OEF a Generadores que Representan Plantas o Unidades de Generación con Períodos de Construcción Superiores al Período de Planeación de la Subasta. Modificado por la Resolución CREG019 de 2008, CREG040 de 2008, CREG056 de 2008, CREG057 de 2008, CREG139 de 2011 y CREG183 de 2011.

[6] CREG, Resolución CREG 071 de 2006. Por la cual se adopta la metodología para la remuneración del Cargo por Confiabilidad en el Mercado Mayorista de Energía. Anexo 12. Reglamento de la Subasta de Sobre Cerrado para Participantes con Plantas y/o Unidades de Generación con Períodos de Construcción Superiores al Periodo de Planeación de la Subasta del Cargo por Confiabilidad (GPPS). Modificado por la Resolución CREG019 de 2008, CREG040 de 2008, CREG056 de 2008, CREG057 de 2008, CREG139 de 2011 y CREG183 de 2011.

[7] CREG, Resolución CREG 071 de 2006. Por la cual se adopta la metodología para la remuneración del Cargo por Confiabilidad en el Mercado Mayorista de Energía. Anexo 3. Cálculo de la Energía Firme para el Cargo por Confiabilidad. Modificado por la Resolución CREG079 de 2006, CREG061 de 2007, CREG085 de 2007, CREG 153 de 2011.

[8] CREG, Resolución CREG 071 de 2006. Por la cual se adopta la metodología para la remuneración del Cargo por Confiabilidad en el Mercado Mayorista de Energía. Artículo 43. Energía de Referencia para el Mercado Secundario.

[9] CREG, Resolución CREG079 de 2007. Por la cual se adicionan, aclaran y modifican algunas disposiciones de la Resolución CREG-071 de 2006. Reglamento del Mercado Secundario del Cargo por Confiabilidad.

[10] CREG, Resolución CREG085de 2007. Por la cual se modifican, aclaran y adicionan disposiciones de la Resolución CREG-071 de 2006 y se dictan otras normas, sobre el Cargo por Confiabilidad. Capitulo Pruebas de Disponibilidad. Modificada por la Resolución CREG138 de 2008, CREG177 de 2008, CREG138 de 2009, CREG148 de 2010, CREG138 de 2012, CREG154 de 2013.

[11] CREG, Resolución CREG063de 2010. Por la cual se regula el anillo de seguridad del Cargo por Confiabilidad denominado Demanda Desconectable Voluntariamente. Modificada por la Resolución CREG203 de 2013.

[12] CREG, Resolución CREG139 de 2011. Por la cual se modifica la Resolución CREG 071 de 2006 y se dictan algunas disposiciones sobre la subasta para la asignación de Obligaciones de Energía Firme del Cargo por Confiabilidad. Artículo 1. Modificación de la definición de Planta y/o Unidad de Generación Nueva contenida en el artículo 2 de la Resolución CREG 071 de 2006. 